\title{
A Large Jackstone Calculus Incidentally Detected on CT Examination: A Case Report With Literature Review
}

\author{
Barbara Brogna ${ }^{\mathrm{a}, \mathrm{b}, \mathrm{c}}$, Federica Flammia ${ }^{\mathrm{b}}$, Francesco Ciriaco Flammia ${ }^{\mathrm{b}}$, \\ Ugo Flammia ${ }^{b}$
}

\begin{abstract}
A rare case of Jackstone calculus in the bladder that was incidentally detected on a computed tomography (CT) examination in a patient with non-specific abdominal pain is described. Jackstone calculus represents a rare entity characterized by a central dense core with peripheral projections that resembles a children's toy. It is important to recognize this entity because it is conducive to fragmentation through lithotripsy. Jackstone calculi are usually described in veterinary literature, but they are rare in humans. In human patients, their formations are caused by urinary obstructions.
\end{abstract}

Keywords: Jackstone calculus; Stone mineral compositions; Urinary obstruction; Imaging

\section{Introduction}

Jackstone calculi are urinary stones with a characteristic shape that reflects their mineral compositions. These types of stone are commonly described in the veterinary literature with a high occurrence in dogs and are made up of silica compositions. They are rarely found in humans and are mainly composed of calcium oxalate dehydrate [1-4]. They may be found in the urinary bladder but even more rarely in the upper urinary tract. There are less than 20 cases of Jackstone calculi in human patients.

\section{Case Report}

A 84-year-old man with cognitive impairment and non-specific abdominal pain came to our observation. Relatives stated that

Manuscript submitted October 1, 2018, accepted October 9, 2018

anstitution of Radiology at 'University of Study of Campania, Luigi Vanvitelli', piazza Miraglia 2, Napoli 80138, Italy

bRadiological Center CEDIR, via Cristofaro Colombo n 10, Grottaminarda 83035, Avellino, Italy

${ }^{\mathrm{c}}$ Corresponding Author: Barbara Brogna, Institution of Radiology at 'University of Study of Campania, Luigi Vanvitelli', piazza Miraglia 2, Napoli 80138, Italy. Email: barbara.brogna@unicampania.it

doi: https://doi.org/10.14740/wjnu372 the man had suffered from benign prostatic hypertrophy for many years and that there was also a history of chronic renal failure and diverticulosis. A month earlier the patient had an episode of macroscopic hematuria. Hematological, microbiological and biochemistry examinations revealed no abnormalities, but confirmed only high creatinine levels. An abdominal computed tomography examination (CT) without contrast was also requested to rule out diverticulitis. The study was performed on a Philips Brilliance CT 16-slice scanner and was completed by multiplanar (MPR) (Fig. 1), 3D maximum intensity projection (MIP) and volume rendering (VR) reconstructions (Fig. 2). A stone of $3 \times 2.4 \times 3.2 \mathrm{~cm}$ with a central dense core and peripheral and speculated projections was visible in the poorly distended bladder. The appearance of the stone was compatible with a Jackstone calculus (Fig. 3). It was located between the bladder body and the dome (Fig. 1a, b). At this point, the bladder walls were also thickened (Fig. 1a). Other little stones with regular appearances were also visible at the part of the bladder base. The patient's prostate was enlarged, measuring $5.7 \times 5 \times 5.3 \mathrm{~cm}$ (Fig. 1b).

The patient refused further treatment.

\section{Discussion}

There are a variety of characteristics for different stone types. A Jackstone calculus is a subtype of urinary calculus characterized by a speculated appearance with a dense core that resembles the six-pointed toys called jacks $[1,4]$. It sometimes referred to as a mulberry stone because of its mamillated appearance [5]. The first studies on urinary calculus composition occurred in the 18th century [6] and the first description of a Jackstone calculus was made by Everidge in 1927 [7]. Calcium oxalate is the most common component of urinary calculi. Pure calcium oxalate monohydrate stones typically are homogenously opaque with smooth margins and appear black, whereas those composed of calcium oxalate dehydrate, such as a Jackstone calculus, tend to be irregular and yellow. They are typically very crystalline in nature. Jackstone calculi with silica compositions are also described in literature. Human silica urolithiasis is estimated to occur in $0.02 \%$ of all human urinary stones and generally is associated with several years of magnesium trisilicate intake [1]. Canine Jackstone calculi are usually composed of silica. Risk factors for Jackstone calculus formation include prostatic diseases, previous lower urinary tract surgery, metabolic abnormalities, upper urinary tract calculi, foreign bodies in the 


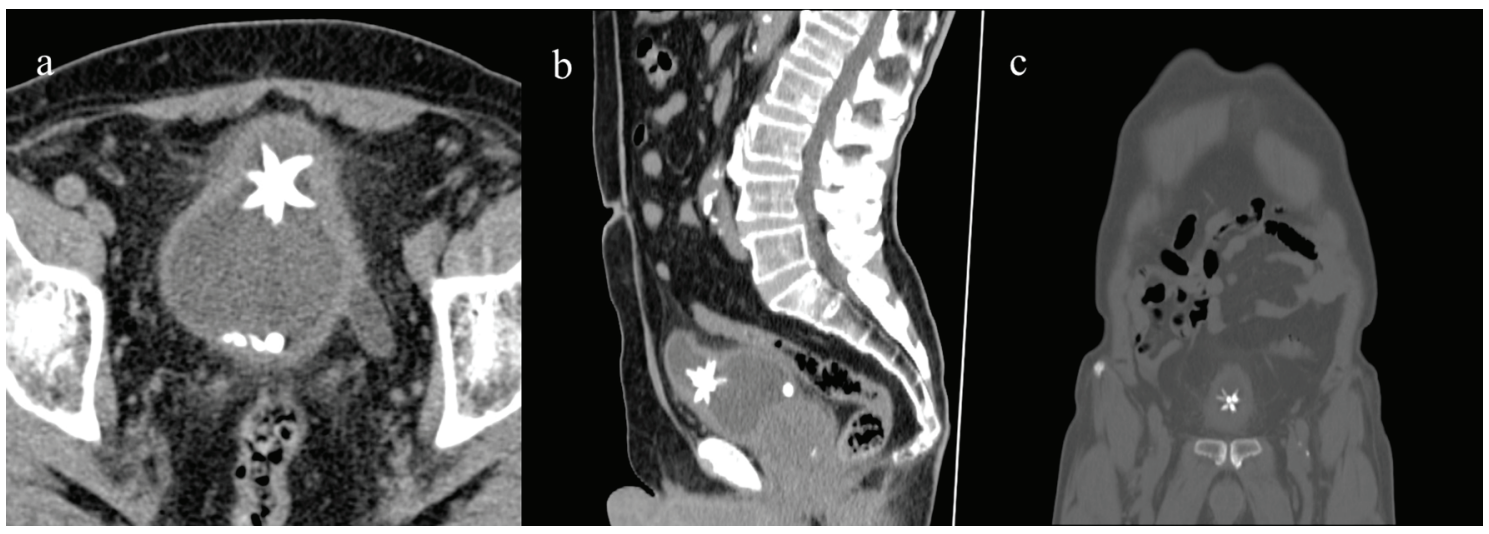

Figure 1. Axial CT showing the Jackstone calculus between the bladder dome and the body with adjacent wall thickness. Other calculi with regular appearances were also visible at the bladder base (a), MPR reconstruction on sagittal (b) and coronal plane (c).

bladder, spinal cord injuries, transplant surgery, dietary factors, dehydration and some medications such as tosuflaxacina and triamterene [1, 3, 4, 8]. However, bladder outlet obstruction remains the most common cause of bladder calculi in adults. The clinical presentation varies from asymptomatic patients to suprapubic pain, dysuria, intermittency, frequency, hesitancy, nocturia to urinary retention. The discomfort is often aggravated by sudden movements and exercise. Complications include chronic bladder irritation, chronic urinary tract infections, fistulas and urethral obstruction. Pericystitis can become chronicle which may result in the adherence of the bladder to the adjacent pelvic fat. Bladder perforation is a rare complication. In our case, the enlarged prostate contributed to bladder stasis and to the growth of Jackstone calculus which caused bladder parietal thickened and chronic irritations. The growth of stones is usually regulated by modulators and/or a deficiency in crystallization inhibitors [6]. However, the typical Jackstone calculus makes contact with the wall only at the peaks of the projections where the mucoprotein and precipitated apatite slowly come off, allowing more calcium oxalate to grow in its place. Due to this, the stone grows only at the tips, producing the speculated jackstone shape, according to Hinman in 1979 [1, 2, 9]. The Jackstone appearance can be easily detected on an ultrasound or a CT examination [9].

The CT evaluation is the most accurate radiological tool. The analysis of Hounsfield unit (HU) makes it possible to observe the homogeneity or heterogeneity structure of a stone and is also able to give the precise size with the use of MPR. In a dual-energy CT, materials can be differentiated by exposing the tissues to two different X-ray spectra or by using a combi-

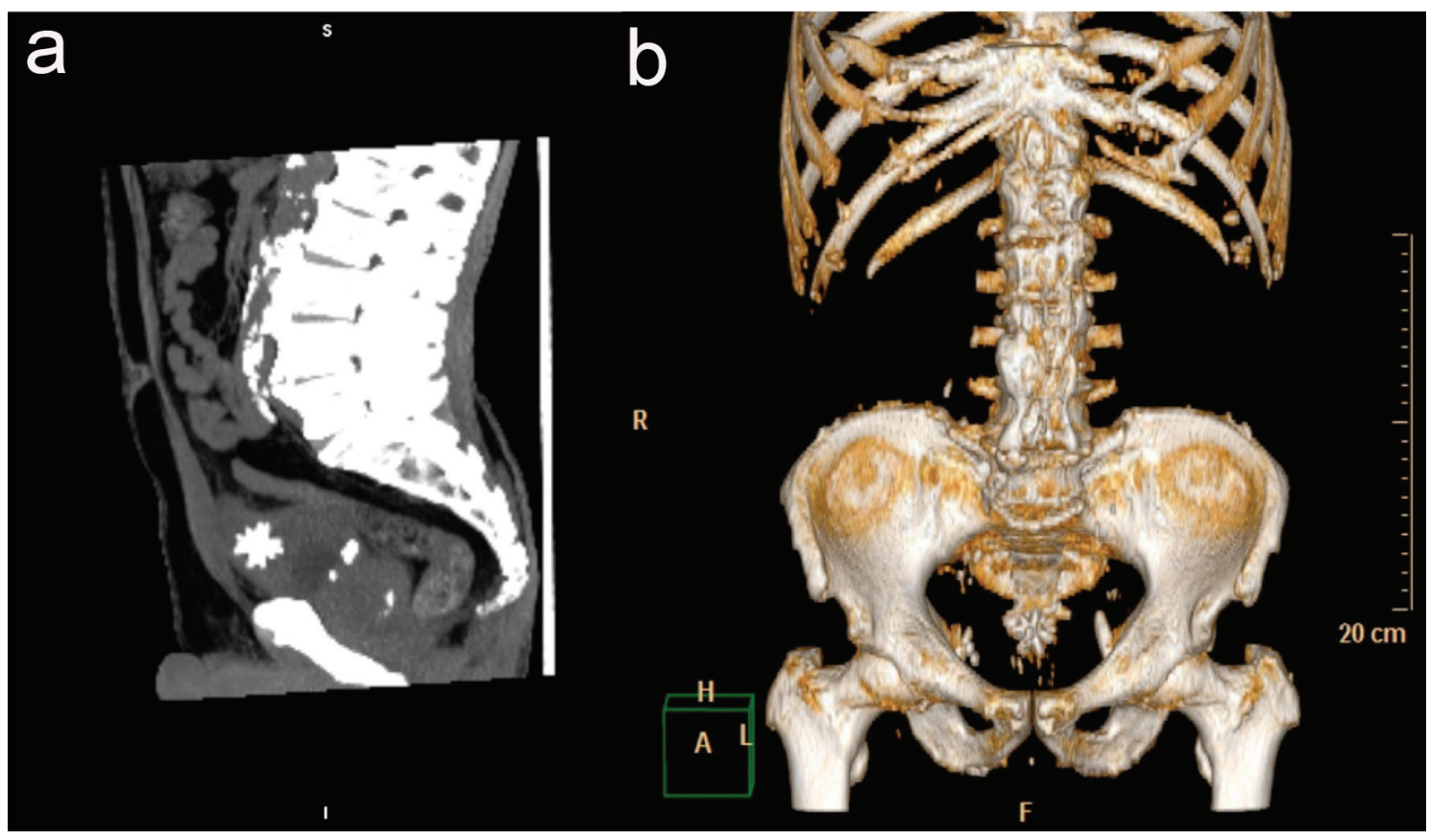

Figure 2. The Jackstone calculus on MIP 3D reconstruction on sagittal plane (a) and on VR (b). 


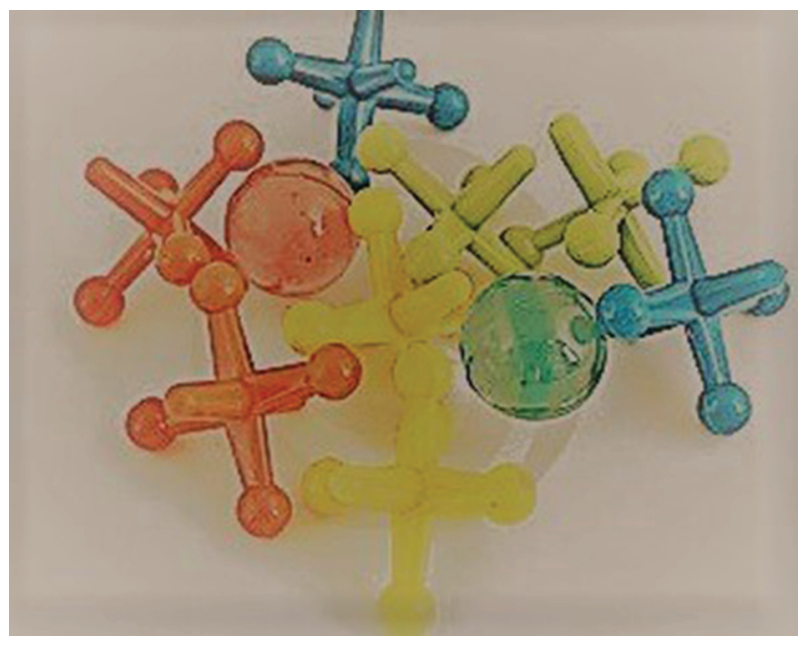

Figure 3. Picture of six-point Jack's toys.

nation detector with two different energy ranges [10].

Stone characteristics are helpful in determining treatment strategies because those characterized by heterogeneous structures are more sensitive to therapeutic fragmentation.

Therefore, it is important to recognize the characteristic shape of Jackstone calculi for therapeutic implications. Calculi made up of calcium oxalate dehydrate are particularly susceptible to fragmentation through lithotripsy. On the other hand, calcium oxalate monohydrate calculi are characterized by a more homogeneous and harder structure. The treatment includes the resolution of all of the causes of bladder obstruction such as benign prostatic hypertrophy (BPH). Treatment of BPH after medical therapy fails involves surgical techniques that include transurethral resection of the prostate, laser prostatectomy, transurethral incision of the prostate, transurethral microwave therapy, transurethral needle ablation and an open prostatectomy [11].

\section{Conclusions}

There are a variety of stone subtypes. It is important to recognize the characteristic shape of the urinary calculi in the bladder on radiological tools for treatment strategies.

\section{Conflict of Interest}

The authors do not have any disclosures to report.

\section{Financial Support}

No funding was received for the publication.

\section{Ethical Approval}

All procedures performed in the studies involving human participants were in accordance with the ethical standards of the institutional and/or national research committee and with the 1964 Helsinki Declaration and its later amendments or comparable ethical standards. For this type of the study, formal consent is not required. Informed consent was obtained from the patient.

\section{References}

1. Perlmutter S, Hsu CT, Villa PA, Katz DS. Sonography of a human jackstone calculus. J Ultrasound Med. 2002;21(9):1047-1051.

2. Roose R, Feyaerts F. Jackstone calculus: a spiky cause of haematuria. J Belg Soc Radiol. 2018;102(1):37.

3. Singh KJ, Tiwari A, Goyal A. Jackstone: A rare entity of vesical calculus. Indian J Urol. 2011;27(4):543-544.

4. Subasinghe D, Goonewardena S, Kathiragamathamby V. Jack stone in the bladder: case report of a rare entity. BMC Urol. 2017;17(1):40.

5. Dyer RB, Chen MY, Zagoria RJ. Classic signs in uroradiology. Radiographics. 2004;24(Suppl 1):S247-280.

6. Grases F, Costa-Bauza A, Prieto RM, Saus C, Servera A, Garcia-Miralles R, Benejam J. Rare calcium oxalate monohydrate calculus attached to the wall of the renal pelvis. Int J Urol. 2011;18(4):323-325.

7. Everidge J. Jackstone Calculi. Proc R Soc Med. 1927;20(5):717-718.

8. Rivell CG, Coren JS. Onesies, Twosies, not a game of Jackstones. Osteopathic Family Physician. 2012;4(3):8890.

9. Sweeney AP, Dyer RB. The "jackstone" appearance. Abdom Imaging. 2015;40(7):2906-2907.

10. Fornaro J, Leschka S, Hibbeln D, Butler A, Anderson N, Pache G, Scheffel H, et al. Dual- and multi-energy $\mathrm{CT}$ : approach to functional imaging. Insights Imaging. 2011;2(2):149-159.

11. Edwards JL. Diagnosis and management of benign prostatic hyperplasia. Am Fam Physician. 2008;77(10):14031410. 